\title{
EXPERIMENTAL STUDIES OF THE CENTRAL NERVOUS SYSTEM RELATED TO ANAESTHESIA : IV. EFFECTS OF PENTOBARBITAL PLACEMENT IN CAUDATE NUCLEUS
}

\author{
K. M. LeIGHTON, M.B., F.R.C.P.(C), and \\ LEONARD C. JENKINS, B.A., M.D., C.M., F.R.C.P.(c) ${ }^{*}$
}

The STATE of AWARENess is known to have a functional relationship with the brain stem reticular formation. This follows the work of Moruzzi and Magoun. ${ }^{1}$ The concept of the ascending (reticular activating) fibres arising from the reticular formation and influencing widespread central nervous system centres is illustrated in Figure 1. It is known that thalamocortical traffic is multidirectional. ${ }^{1}$

The state of awareness is characterized in electroencephalographic recordings obtained from the cerebral cortex of man and experimental animals by the pattern of activation. ${ }^{1}$ This consists of fast, low amplitude waves in the EEG tracing. Sleep, on the other hand, is associated with the EEG pattern of deactivation which is characterized by higher amplitude and slower electrical recordings. During the production and maintenance of anaesthesia, there is a graded pattern of fast activity progressing through slower activity to increasingly prolonged periods of suppression (Fig. 2). ${ }^{2}$

It has not proved possible to produce deep sedation, or marked cortical deactivation, by the local injection of pentobarbital into the brain stem reticular formation. Therefore, a search was instituted for other subcortical sites which might prove more sensitive to the action of this drug.

\section{METHOD}

The technique of stereotaxic placement and utilization of subcortical recording cannulae has been described elsewhere. ${ }^{3-5}$ The accuracy of electrode placement into subcortical sites was confirmed by subsequent histological examination. Pentobarbital $(500 \mathrm{mg} / \mathrm{ml})$ was injected into the sites mentioned below. This solution has the following characteristics: $\mathrm{pH} 9.0$ and $0.004 \mathrm{mosm} / \mu$ litre.

The sites injected bilaterally were: amygdala, hippocampus, ventroposterolateral thalamic nucleus, centro-median thalamic nucleus, posterior hypothalamic nuclei, and head of caudate nucleus. Doses of from 1,000 to $3,000 \mu \mathrm{g}$ were employed. Control injections of sodium chloride, adjusted for $\mathrm{pH}$ and osmolarity, were also administered.

The animals were placed in an observation box with a one-way viewing window. They were connected to a Grass electroencephalograph via a skull plug (Cannon) and a connecting wire. The cats were fully mobile within the observation box. At intermittent periods, auditory stimuli were applied. These took the

'From the Departments of Pharmacology and Surgery (Division of Anaesthesiology), University of British Columbia, Vancouver. 


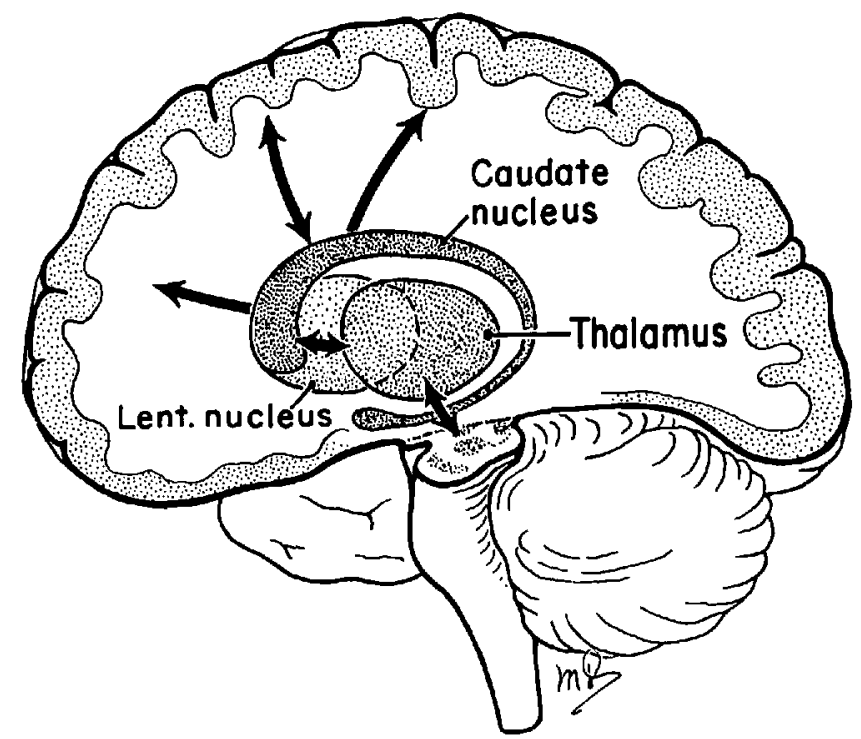

Ficure 1. Diagram illustrating the concept of the ascending (reticular activating) fibres arising from the reticular formation of the mesencephalon and having widespread multidirectional connections and influences on central nervous system structures, including the caudate nucleus and their function (lent. nucleus-lenticular nucleus).

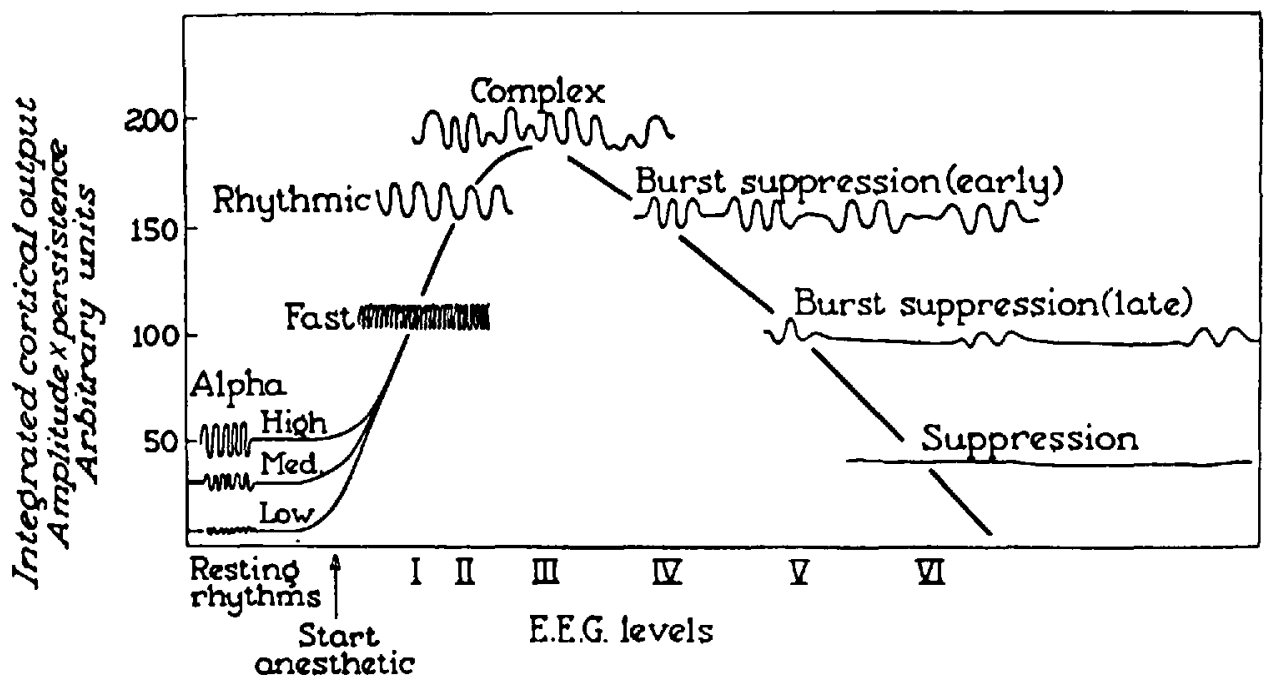

FIGURE 2. Diagram of average EEG pattern changes during anaesthesia (with kind permission of the authors and publisher, from Martin et al. ${ }^{2}$ ).

form of a loud tap on the viewing box window, an electrically produced click, or a whistle blast.

\section{Results AND Discussion}

Figures 3, 4, 5, 6, and 7 are electrical recordings obtained from the experimental animals prepared as previously described. Recordings are bilateral, from 
I Fr CX

R Fr CX

$L C D$ a

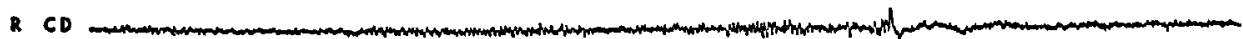

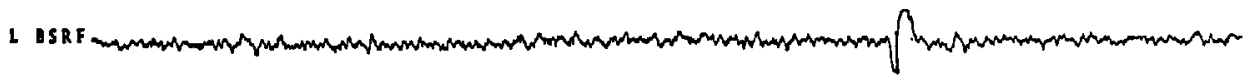

R BSRF

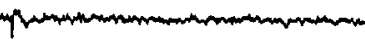

$t$

stim.

$\rightarrow 50 \mu \mathrm{V}$

1 sec

Frgune 3. Control patterns in left frontal cortex ( $\mathrm{L} \mathrm{Fr} \mathrm{Cx}$ ), right frontal cortex ( $\mathrm{R} \mathrm{Fr} \mathrm{Cx}$ ), left head caudate nucleus (LCD); right head caudate nucleus (RCD), left brain stem reticular formation (LBSRF), and right brain stem reticular formation (RBSRF). (See text for discussion.)

1 Fr Cx

R $\mathrm{Fr}$ Cl

1 CO M 1500

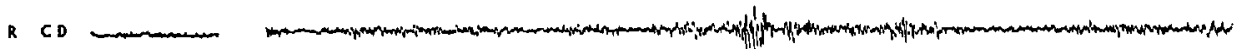

PB 1500 ug

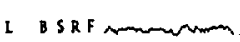

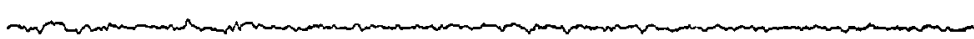

R BSRF

$$
\underset{1 \text { sec }}{50 \mu \mathrm{V}}
$$

Figure 4. Six minutes after pentobarbital $(1500 \mu \mathrm{g})$ was placed into both caudate nuclei (heads). 
the frontal cortices ( $L$ Fr Cx and R Fr Cx), caudate nuclei ( $L C D$ and $\mathrm{RCD}$ ), and the brain stem reticular formation (LBSRF and RBSRF).

Figure 3: control. Rapid, low amplitude activity is apparent, particularly in the cortical recordings. Some spontaneously occurring periods of relative deactivation can be observed. An auditory stimulus (stim.) evokes an electrical response and is followed by a period of renewed activation.

Figure 4. Pentobarbital $(1500 \mu \mathrm{g})$ is injected into both caudate nuclei (heads). The recordings on the left are the control patterns. The gap in the recording represents a time interval of six minutes. Deactivation in the cortical and caudate recordings is particularly evident and is more marked than that which was observed spontaneously prior to the pentobarbital injection.

Figure 5. Pentobarbital 12 minutes after injection. In this recording an auditory stimulus (stim.) can be seen to evoke an electrical response and a short-lived episode of slight activation in the electrical recordings obtained from the cortices. As far as the recordings obtained from the caudate nuclei and the brain stem reticular formation are concerned, there is virtually no response to be observed.

Figure 6. Pentobarbital 16 minutes after injection. The pattern is one of marked deactivation. In this particular cat, however, the recording obtained from the right brain stem reticular formation shows mild deactivation only. An auditory response is not evoked and there is no following period of activation, in cortices, caudate nuclei, or brain stem reticular formation tracings.

Figure 7. Pentobarbital 24 minutes after injection. Spontaneous activation appears in all the previously deactivated areas and, once more, an auditory stimulus evokes an electrical response. although still less than the control pattern.

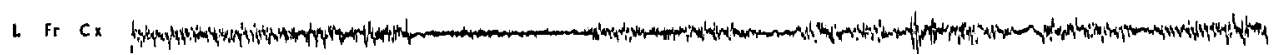

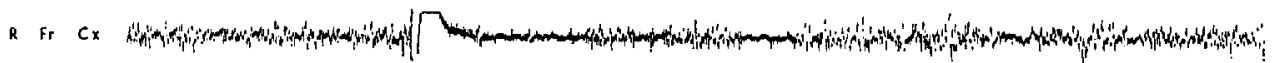
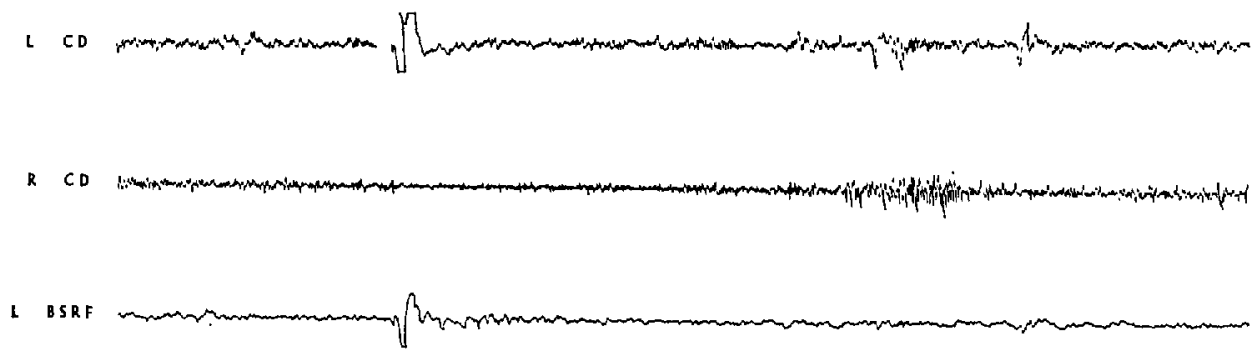

R BSRF

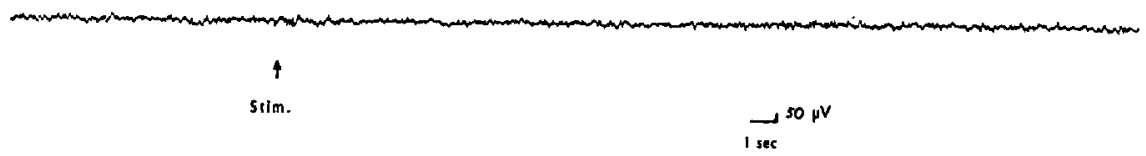

Figure 5. Twelve minutes after pentobarbital was placed into both caudate nuclei (heads). 
L FE CX

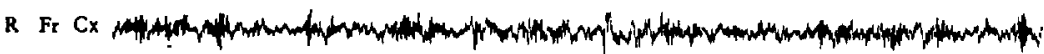

L CD

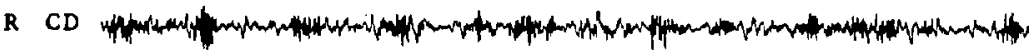

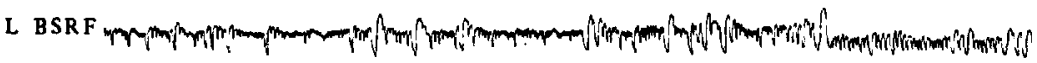

R BSRF

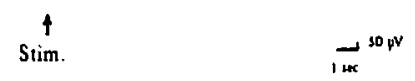

Figure 6. Sixteen minutes after pentobarbital was placed into both caudate nuclei (heads).

In all instances, behavioural sedation accompanied EEG deactivation. Thus, following the application of pentobarbital into the sites designated above, only that administered to the head of the caudate nucleus produced consistent, reproducible behavioural and electroencephalographic evidence of sedation in the presence of repetitive stimuli. This effect was obtained following the injection of $1500 \mu \mathrm{g}$ of pentobarbital. Within four to six minutes, cortical deactivation was obtained, and 15 minutes after the injection, the animals became unresponsive to

L Fr CX

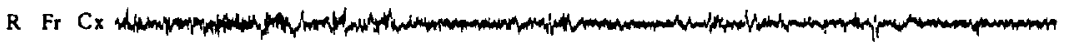

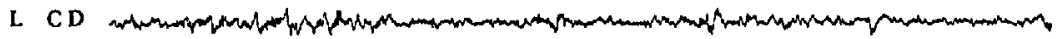

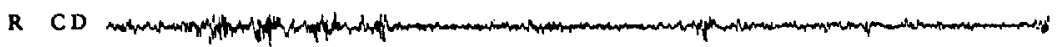

L $B S R F$

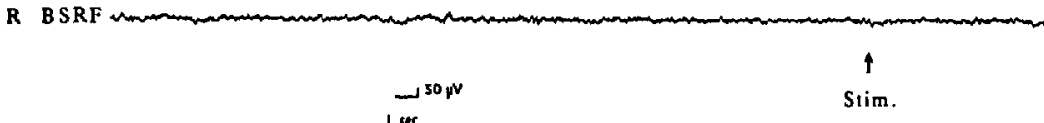

Figure 7. Twenty-four minutes after pentobarbital was placed into caudate nuclei (heads). 
auditory stimuli. After 25 minutes, spontaneous activation of the cortical encephalogram returned and the animals were behaviourally awake.

This, perhaps, is in accord with the reports of other workers, who have found a correlative relationship to exist between the caudate nucleus and behavioural and electrical activity in experimental animals. ${ }^{6-9}$ For instance, Kitsikis and asso$\operatorname{ciates}^{\theta}$ have demonstrated that spindle-like synchronized waves and behavioural sedation (not unlike light barbiturate sedation) can be elicited by stimulating the head of the caudate nucleus in the monkey and cat. This was shown not to be due to internal capsule influence and indicated that the caudate nucleus participated in synchronizing cortical activity.

It would appear, from these results, that the head of the caudate nucleus in the cat is more sensitive to the action of locally applied barbiturates than other subcortical sites which have been examined in this study.

\section{SUMMARY}

Deep sedation results, in cats, when pentobarbital is placed in the head of caudate nucleus through precise stereotaxically implanted recording cannulae. This sedation persists in the presence of a variety of auditory stimuli, as observed by a lack of behavioural arousal and alertness and the persistence of an EEG and subcortical correlate of deactivation.

Following the application of pentobarbital into amygdala, hippocampus, ventropostero-lateral thalamic nucleus, centro-median thalamic nucleus, and posterior hypothalamic nuclei, there was no persistent behavioural or electroencephalographic evidence of sedation in the presence of repetitive stimuli.

\section{ACKNOWLEDGMENTS}

Financial assistance for this study was provided by MRC grant $68-2145$, by grants-in-aid from $60-0050$, and by Burroughs Wellcome Laboratories, Poulenc Ltd., Ayerst McKenna Laboratories Ltd., and Abbott Laboratories.

\section{REFERENCES}

1. Moruzzi, G. \& MAgoun, H. W. The Brain Stem Reticular Formation and Activation of the EEG. Clin. Neurophysiol. 1: 455 (1949).

2. Martin, J. T.; Faulconer, A. JR; \& Bickford, R. G. Review Article: Electroencephalography in Anaesthesiology. Anaesthesiology. 20: 359 (1959).

3. Jenkins, L. C.; Ling, G. M.; Foulks, J. G.; \& Penrose, G. S. Experimental Central Nervous System Studies Related to Anaesthesia: Clinical Implications: I. Anaesthesia and the Brain Stem Reticular Formation. II. Effect of Reduced Sensory Inflow on Anaesthetic Requirements. Canad. Anaesth. Soc. J. 10: 634 (1963).

4. Yamaguchi, N.; Ling, G. M.; \& Marczynski, T. J. Recruiting Responses Observed during Wakefulness and Sleep in Unanaesthetized Chronic Cats. EEg Clin. Neurophysiol. 17: 246 (1964).

5. Munkoe, J. P.; Jenkins, L. C.; \& Ling, G. M. Experimental Central Nervous System 
Studies Related to Anaesthesia: Clinical Implications. III. Effects of Muscle Relaxants on Sensory Inflow. Canad. Anaesth. Soc. J. 13: 109 (1966).

6. MacLennan, H. \& York, D. H. Some Behavioural Effects of Stimulation of the Caudate Nucleus in Unrestrained Cats. Canad. J. Physiol. Pharmacol. 42: 329 (1964).

7. MacLennan, H. \& York, D. H. Cholinergic Mechanisms in the Caudate Nucleus. J. Physiol. 187: 163 (1966).

8. Stevens, J. Stimulation of Caudate Nucleus: Behavioural Effects of Chemical and Electrical Excitation. Arch. Neurol. 4: 47 (1961).

9. Krtsixis, A.; Honvath, F. E.; \& Rougeul, A. Synchronized Spindle Activity Elicited in the Cortex of the Monkey by Basal Ganglia Stimulation. EEG Clin. Neurol,, 25: 160 (1968). 